Document downloaded from:

http://hdl.handle.net/10251/123460

This paper must be cited as:

Morant, M.; Llorente, R. (2018). Performance Analysis of Carrier-Aggregated Multi-Antenna $4 \times 4$ MIMO LTE-A Fronthaul by Spatial Multiplexing on Multicore Fiber. Journal of Lightwave Technology. 36(2):594-600. https://doi.org/10.1109/JLT.2017.2786582

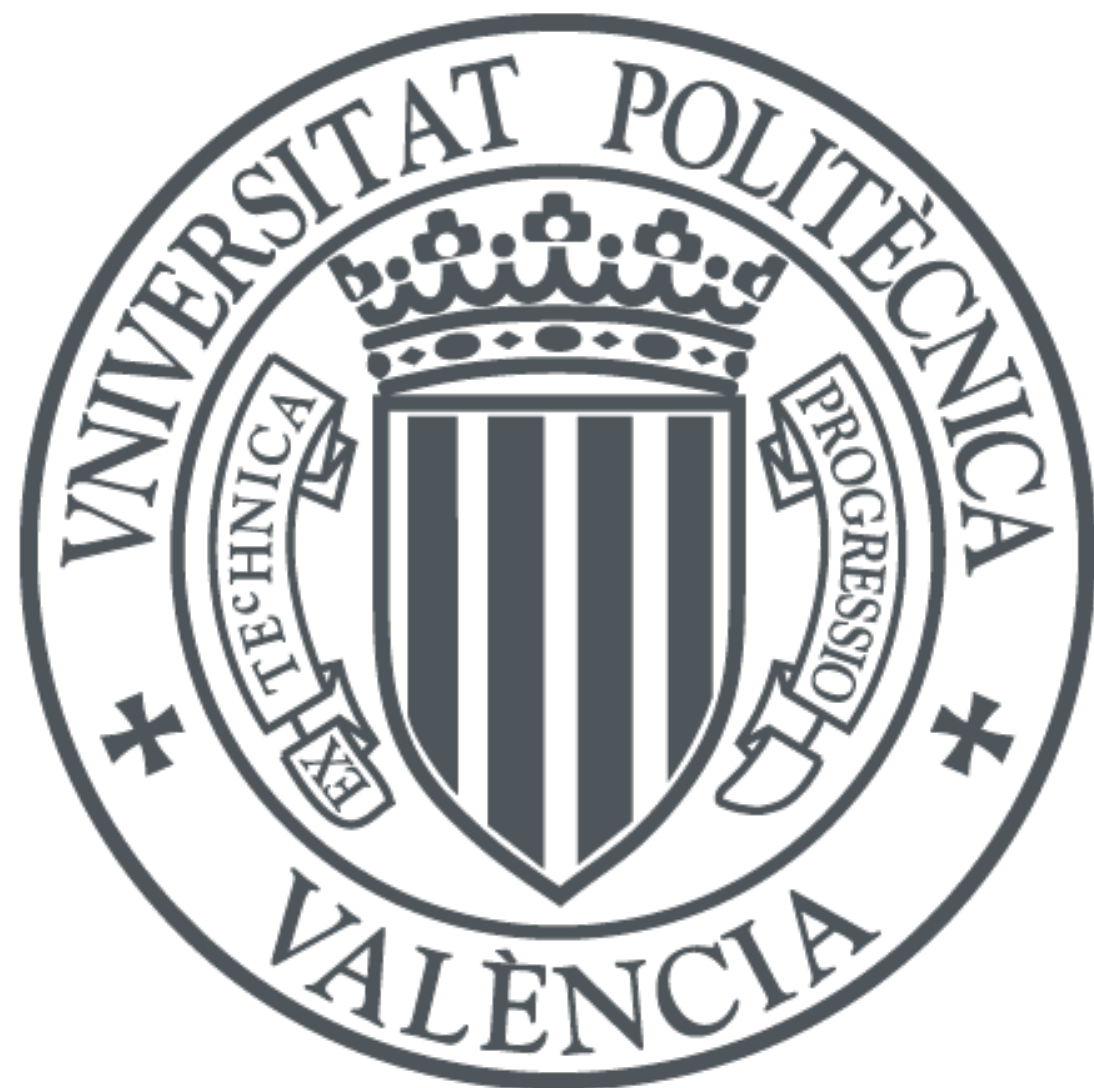

The final publication is available at

http://doi.org/10.1109/JLT.2017.2786582

Copyright Institute of Electrical and Electronics Engineers

Additional Information

(C) 2018 IEEE. Personal use of this material is permitted. Permissíon from IEEE must be obtained for all other uses, in any current or future media, including reprinting/republishing this material for advertisíng or promotional purposes, creating new collective works, for resale or redistribution to servers or lists, or reuse of any copyrighted component of this work in other works. 


\title{
Performance Analysis of Carrier-Aggregated Multi-Antenna 4×4 MIMO LTE-A Fronthaul by Spatial Multiplexing on Multicore Fiber
}

\author{
Maria Morant, Member, IEEE, and Roberto Llorente, Member, IEEE
}

\begin{abstract}
In this work we propose and evaluate experimentally the performance of multi-antenna LTE-Advanced (LTE-A) systems implementing MIMO space division multiplexing on multicore fiber, compared to single-antenna SISO transmissions. Fully standard 3GPP LTE-A cellular signals are transmitted with MIMO and Carrier Aggregation in radio-overfiber over a four-core fiber in different configurations. The processing capabilities of in-built 3GPP MIMO processing are evaluated experimentally in two-antenna and four-antenna LTE-A configurations and compared with single-antenna SISO performance. The robustness of 3GPP MIMO processing is analyzed over different optical paths in a 4-core fiber and the optical power margin available between the four optical paths is calculated for each configuration. Finally, the transmission performance of carrier-aggregated LTE-A signals is evaluated in the four-antenna system implementing $4 \times 4$ MIMO spatial multiplexing with different carrier separation and center frequency configurations, including regulated cellular frequencies of FDD bands 7 and 20.
\end{abstract}

Index Terms - Multicore fiber, space division multiplexing, MIMO systems, carrier aggregation, radio-over-fiber

\section{INTRODUCTION}

W IRELESS data traffic explosion in the last decade has led to higher data rate requirements for next-generation wireless cellular networks which are expected to bring Gbps per mobile user by 2020 [1]. It is expected that mobile broadband network growth reaches 10 billion subscribers by the year 2020, which may result in a compound annual growth rate (CAGR) of $5.4 \%$ between 2014 and 2020 [2]. The traffic growth related with this CAGR implies high network densification. To achieve this, several technologies are proposed to be integrated in the next-generation networks

Manuscript received July 1, 2017, reviewed November 2, 2017, accepted December 17, 2017. This research was supported in part by Spain National Plan MINECO/FEDER UE TEC2015-70858-C2-1-R XCORE and RTC2014-2232-3 HIDRASENSE. The work of M. Morant supported by UPV postdoc PAID-10-16 program.

M. Morant and R. Llorente are with the Nanophotonics Technology Centre, Universitat Politècnica de València, 46022 Valencia, Spain, (e-mail: mmorant@ntc.upv.es; rllorent@ntc.upv.es). including cloud radio access networks (C-RAN) and dense heterogeneous networks (HetNets) employing massive multiple-input-multiple-output (M-MIMO) and Carrier Aggregation [3]. C-RAN architecture is the most promising approach for the massive deployment of small cells with reduced capital and operational expenses thanks to the complexity reduction of conventional cell sites [4]. C-RAN main advantages are: (i) the capability of advanced inter-cell coordination (as the baseband processing for the radiowave to/from different antennas is centralized in the same location), and (ii) the reduction of the size antenna-site equipment and footprint [5]. The C-RAN mobile base-station equipment comprises a baseband unit (BBU) and several remote radio heads (RRHs) as represented in Fig. 1, where the BBU is responsible for radio frequency functionalities while the RRHs have reduced power-consumption requirements [5]. Currently, MIMO and Carrier Aggregation techniques are developed in several wireless standards, such as IEEE 802.11 WLAN, IEEE 802.16 WiMAX and 3GPP LTE-Advanced (LTE-A).

There are two factors driving the capacity increase in LTE-A: First, the use of Carrier Aggregation, where the overall transmission bandwidth is increased by aggregating several signal carriers, each one known as a component carrier (CC). As depicted in Fig. 1, when Carrier Aggregation is used, each $\mathrm{CC}$ can serve a different cell, with the same or different coverage. Second, the support of massive MIMO improves the spectral efficiency by using a large number of antennas and accommodating dozens of users in the same radio channel [6].

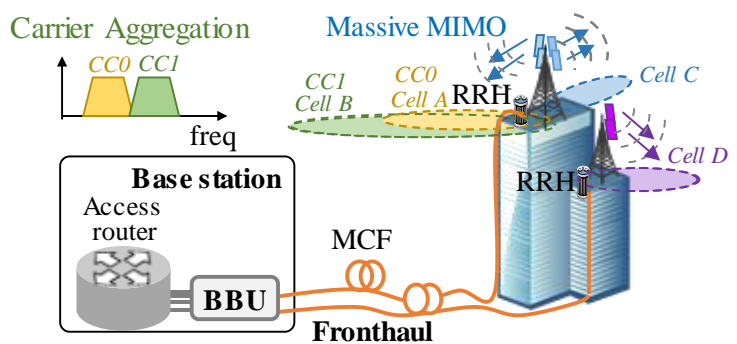

Fig. 1. Fronthaul scenario implementing radio-over-multicore fiber with massive MIMO and Carrier Aggregation 


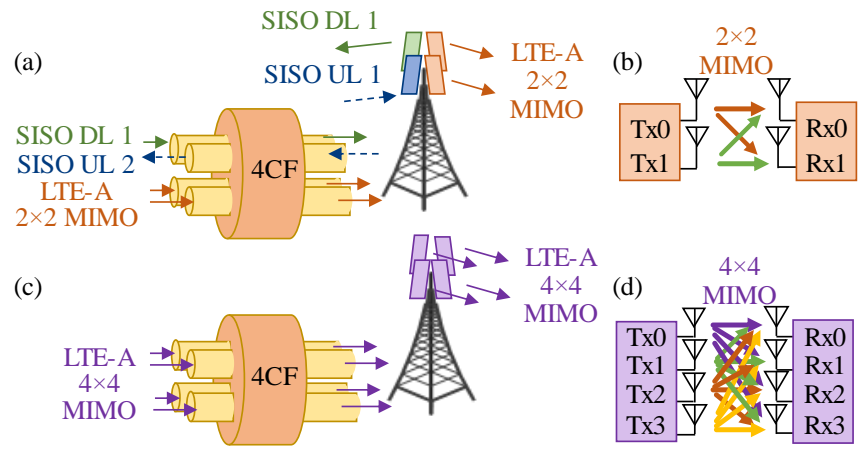

Fig. 2. (a) Proposed core allocation for single- and two-antenna radio-overmulticore fiber provision. (b) Two-antenna system implementing $2 \times 2 \mathrm{MIMO}$ spatial multiplexing (data streams coded in 2 layers). (c) Core allocation for four-antenna radio-over-multicore fiber transmission. (d) Single-user fourantenna system implementing $4 \times 4$ MIMO (data coded in 4 layers)

MIMO is used to increase the overall bitrate through the transmission of two or more different data streams on different antennas [7]. The combined use of Carrier Aggregation and M-MIMO in different degrees [8] defines next-generation 4.5G Pro and 4.9G LTE-A wireless standards [9]. As depicted in Fig. 1, fronthaul antenna systems are usually installed on top of roofs ensuring line-of-sight communication with mobile terminals [1]. In order to provide M-MIMO connectivity, radio-over-fiber (RoF) transmission has been proposed to provide centralized multi-service delivery [5].

As evaluated in the state-of-the-art, MIMO provision requires a specific $\mathrm{RoF}$ architecture as the group of MIMO signals use the same carrier frequency and must be compatible with carrier aggregation in cellular systems. Different solutions have been proposed in the literature, such as: (i) Employing different SSMF links with individual optoelectronic devices for each MIMO signal, which is far too expensive for massive MIMO application [10]. (ii) Assigning different wavelengths to each MIMO stream and implementing wavelength division multiplexing (WDM) [11] which is an expensive (multiwavelength laser sources needed [12]) and complex solution as each stream suffers different chromatic dispersion along the fiber. (iii) Assigning different frequencies to each MIMO stream implementing subcarrier multiplexing (SCM) [13], which requires down/up-conversion at the antenna premises and suffers of carrier suppression along fiber transmission. (iv) Using optical polarization division multiplexing (PDM) [14], which can only multiplex two channels [12]. (v) Implementing mode division multiplexing (MDM) of different MIMO streams over different optical modes, which requires an expensive spatial light modulator [15]. And, finally, (vi) employing space division multiplexing (SDM) over different cores of a multicore fiber (MCF) [16]. The use of SDM in C-RAN based on MCF appears as a suitable and interesting technique to overcome the capacity crunch defined by the expected CAGR in conventional single-core optical systems. Radio-over-multicore fiber can transmit MIMO signals in SDM, taking advantage of the digital signal processing algorithms already in-place in commerciallyavailable 3GPP LTE-A MIMO equipment [7][16]. The proposed architecture can be scaled to massive MIMO deployment scenarios considering large core-count MCF are a viable solution as recently reported [17]. In this case, more data streams could be multiplexed over the MCF cores.

Fig. 2 shows the proposed core allocation for the radioover-multicore fiber transmission of multi-antenna MIMO systems. Fig. 2(a) shows a two-antenna system multiplexing two data streams with $2 \times 2$ MIMO as depicted in Fig. 2(b). Fig. 2(c) shows the transmission of a different data stream in each core of a four-core fiber implementing $4 \times 4$ MIMO to multiplex the data in four different layers and providing almost 4 times the bitrate of a SISO transmission over the same bandwidth. Single-user configuration are evaluated in this work to increase the provided data-rate, although the proposed RoF system could also be applied to multi-user arrangements.

This paper is structured as follows: In Section II, the capability of MIMO processing algorithms embedded in 3GPP LTE-A standard for two-antenna and four-antenna systems is evaluated experimentally and compared with single-antenna SISO transmission. The robustness of the MIMO algorithms to overcome the impairments in a given path is also evaluated experimentally for each configuration. Next, in Section III, the performance of a four-antenna system implementing $4 \times 4$ MIMO is evaluated with carrier aggregation in different frequency bands and with different carrier separation. Finally, in Section IV, the main conclusions are summarized.

\section{Multi-Antenna LTE-A System Performance OVER MULTICORE FIBER}

\section{A. LTE-A signal generation}

Current LTE-A cellular communication standard implements both technologies aforementioned to provide higher user data rates: multi-antenna MIMO transmission and Carrier Aggregation. Implementing MIMO, the same resources can be used in both frequency and time, separated only through use of different reference signals (RS) to be received by several antennas [16]. In this work, we configure the LTE-A signal to implement a single-antenna system or spatial multiplexed systems with two or four antennas. In Carrier Aggregation, the most used configuration comprises contiguous component carriers within the same operating frequency band (also called intra-band contiguous carriers). In 3GPP release R10 and R11, the intra-band contiguous aggregation in frequency division duplex (FDD) defines a maximum aggregated bandwidth of $40 \mathrm{MHz}$ with two CCs maximum. Thus, in order to evaluate the most challenging case, in this study the 3GPP FDD LTE-A generator (wireless test set Keysight E6640A EXM as depicted in Fig. 3) is configured to transmit two aggregated CCs of $20 \mathrm{MHz}$ bandwidth each. The aggregated bandwidth of $40 \mathrm{MHz}$ can be supported with a single transceiver. Each LTE-A $20 \mathrm{MHz}$ CC comprises 100 resource blocks (RB) with 1201 subcarriers for downlink (DL) and 1200 subcarriers for the uplink (UL). This difference in the number of subcarriers is based on the fact that, for DL signals, the DC subcarrier is not transmitted but counted, while UL signals are symmetric about DC and have no DC subcarrier [18]. 


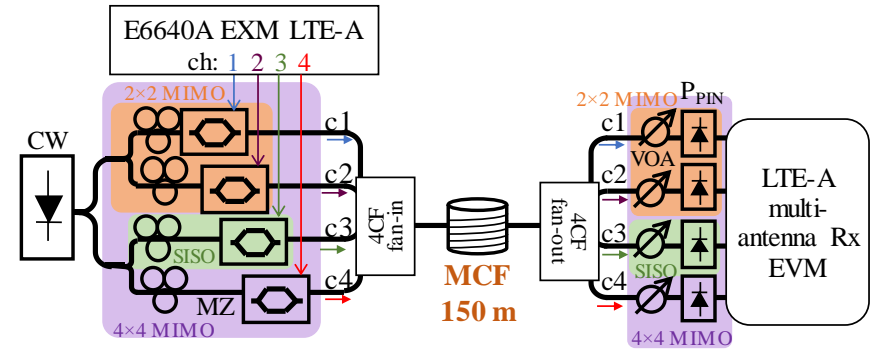

Fig. 3. Experimental setup of the evaluation of a single-antenna, two-antenna and four-antenna LTE-A system implementing SISO, $2 \times 2$ and $4 \times 4$ MIMO spatial multiplexing over MCF respectively

TABLE I

3GPP LTE-A DOWNLINK AND UPLINK PHY CHANNELS

\section{Downlink}

P-SS: Primary synchronization signal S-SS: Secondary synchronization signal PBCH: Physical broadcast channel PCFICH: PHY control format indicator ch PHICH: PHY hybrid ARQ indicator ch PDCCH: Physical downlink control ch C-RS: Cell-specific reference signal PDSCH: Physical downlink shared ch

\section{Uplink}

PUCCH: Primary uplink control channel PRACH: Physical random access channel PUSCH: Primary uplink shared channel
Table 1 summarizes the content of DL and UL LTE-A frames. The DL frame includes the users' information, the physical signals for synchronization -the primary (P-SS) and the secondary synchronization signal (S-SS)-, channel compensation (RS) and control channels. The UL user frame consists of uplink user data (PUSCH) with sounding reference signals (SRS), random-access requests (PRACH) and user control channels (PUCCH) [18].

In this study, the shared user channels (PDSCH in DL and PUSCH in UL) are configured with 16QAM subcarrier mapping using two codewords, which 3GPP recommendation sets the error vector magnitude at $\mathrm{EVM}_{16 \mathrm{QAM}}=12.5 \%$. We configure the wireless test set (Keysight E6640A EXM) to generate two fully standard 3GPP FDD LTE-A CCs of $20 \mathrm{MHz}$ each centered at $f_{c}$ and separated $\Delta f$.

Following the experimental setup depicted in Fig. 3, the $\mathrm{RoF}$ transmission is performed using four Mach-Zehnder (MZ) electro-optical modulators to transport the signals over a continuous wave $(\mathrm{CW})$ at $1555.75 \mathrm{~nm}$. From the four output channels of the wireless test set, channel 1 is defined as the 'master' (which is used to synchronize the different MIMO data streams) while the other output channels are called 'slave'. The data is coded in one, two or four different layers depending on the system under evaluation (single antenna, two-antenna or four-antenna, respectively).

The modulated signals are injected to the MCF using 3D fan-in/fan-outs injecting optical power levels of $-1 \mathrm{dBm}$ into each core. A four-core fiber with mode field diameter of is $8.4 \mu \mathrm{m}$ is employed, with 0.15 numerical aperture and $1410 \mathrm{~nm}$ cut-off wavelength. The RoF transmission is evaluated over $150 \mathrm{~m}$ of 4-core fiber spooled with an average bending radius of $67 \mathrm{~cm}$, in order to evaluate the radio-overmulticore fiber performance with an average inter-core crosstalk of $40.8 \mathrm{~dB}$. This MCF configuration was selected to evaluate the performance with $+10.2 \mathrm{~dB}$ more crosstalk compared with spools of half its radius [7]. At the receiver side, variable optical attenuators (VOAs) are used to evaluate the performance for different power levels at the photodiode (P PIN) keeping the same power level injected to the MCF. The quality of received signals after MCF transmission is evaluated with Keysight Vector Signal Analyzer.

\section{B. Two-Antenna $2 \times 2$ MIMO LTE-A Radio-over-Multicore fiber Transmission Performance}

Fig. 4 shows the experimental setup used to compare the performance of a single-antenna system (SISO) and a twoantenna LTE-A system implementing $2 \times 2$ MIMO spatial multiplexing over MCF. In this section, we evaluate the performance of LTE-A downlink (DL) and uplink (UL) signals with the RoF transmission over MCF and compare it with a $2 \times 2$ MIMO LTE-A signal. In this experiment we generate a fully standard 3GPP LTE-A carrier-aggregated signals comprising two $20 \mathrm{MHz}$ CCs separated $\Delta f= \pm 9.9 \mathrm{MHz}$ in cellular FDD band 7. Thus, the LTE-A DL signal is centered at $f_{c}=2.655 \mathrm{GHz}$ and the LTE-A UL signal is centered at $f_{c}=2.535 \mathrm{GHz}$.

Table 2 reports the measured EVM for a single-antenna SISO LTE-A system in DL and UL configurations measured for $\mathrm{CC} 0$ located at $f_{c}-9.9 \mathrm{MHz}$. In the case of the UL signal, the received EVM quality is measured for the data modulated with 16QAM subcarrier mapping and for the Zadoff-Chu sequence in the demodulation reference signal (PUSCH_DMRS), which corresponds to the constellation points on a circle centered about the origin in Fig. 5(b).

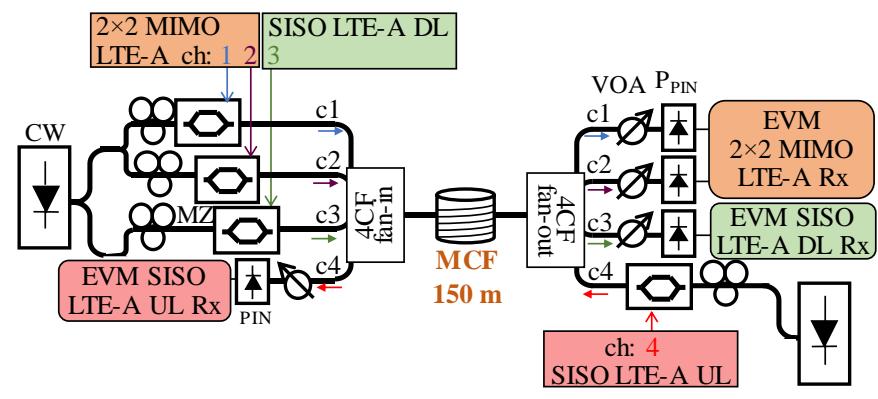

Fig. 4. Experimental setup for the evaluation of two-antenna LTE-A systems including carrier-aggregation in DL $(2 \times 2 \mathrm{MIMO})$ compared with singleantenna LTE-A (SISO) in DL and UL directions.

TABLE II

EXPERIMENTAL RESULTS OF SINGLE-ANTENNA LTE-A TRANSMISSION OVER MCF FOR DOWNLINK AND UPLINK PHY CHANNELS

\begin{tabular}{cc}
\hline SISO DL with $\mathbf{P}_{\mathbf{P I N}}=\mathbf{- 1 0} \mathbf{d B m}$ & SISO UL with P PIN $=\mathbf{- 9} \mathbf{~ d B m}$ \\
P-SS $=11.92 \%$ & PUSCH_DMRS $=2.25 \%$ \\
$\mathrm{~S}-\mathrm{SS}=12.23 \%$ & PUSCH_16QAM $=11.55 \%$ \\
$\mathrm{PBCH}=12.53 \%$ & \\
PCFICH $=13.04 \%$ & \\
PHICH $=10.84 \%$ & \\
PDCCH $=13.8 \%$ & \\
C-RS $=12.13 \%$ & \\
PDSCH $=12.18 \%$ & \\
\hline \hline
\end{tabular}




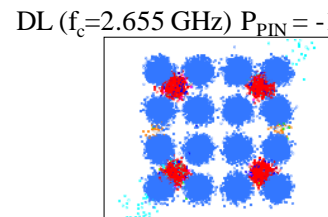

(a)
$\mathrm{UL}\left(\mathrm{f}_{\mathrm{c}}=2.535 \mathrm{GHz}\right) \mathrm{P}_{\mathrm{PIN}}=-9 \mathrm{dBm}$

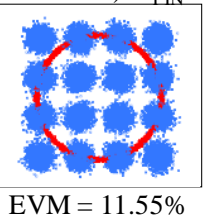

(b)

$\mathrm{EVM}=11.55 \%$

Fig. 5. Received CC0 constellations and EVM for a single-antenna (SISO) LTE-A: (a) DL signal at PPIN $=-10 \mathrm{dBm}$, and (c) UL signal at PPIN $=-9 \mathrm{dBm}$

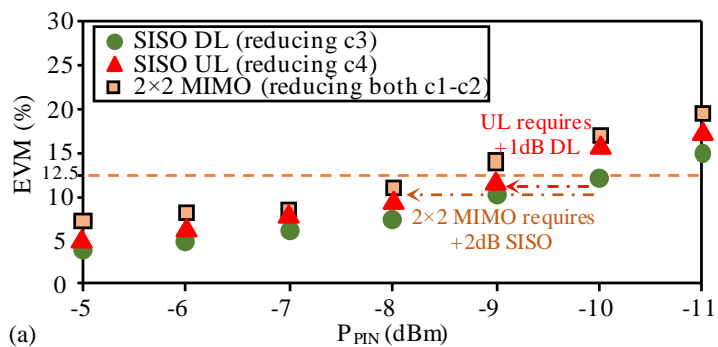

(a)

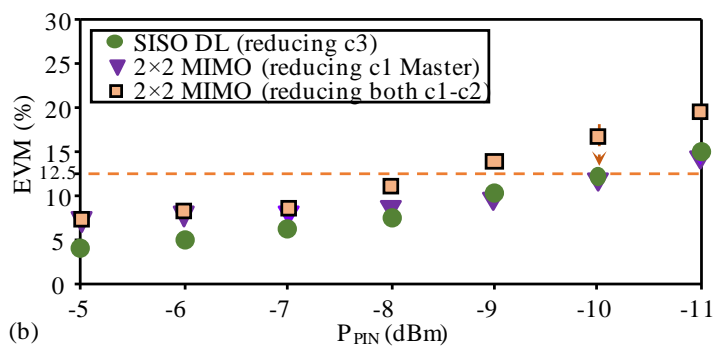

Fig. 6. Measured CCO EVM for LTE-A over multicore transmission vs. received optical power level at the photodiode (PpIN) for (a) single-antenna (SISO) in DL and UL directions and two-antenna $2 \times 2$ MIMO systems. (b) Improvement of a two-antenna system reducing only one of the two optical paths in the $2 \times 2$ MIMO processing.

Fig. 6(a) shows the performance comparison of $2 \times 2 \mathrm{MIMO}$, SISO DL and SISO UL when reducing the optical power level arriving at the photodiode $\left(\mathrm{P}_{\mathrm{PIN}}\right)$. The experimental results point out that the $3 \mathrm{GPP}$ EVM recommendation is met in a single-antenna SISO LTE-A system for $P_{P I N} \geq-10 \mathrm{dBm}$ for DL signals and for $\mathrm{P}_{\mathrm{PIN}} \geq-9 \mathrm{dBm}$ in the case of UL signals. Comparing with the performance of a two-antenna $2 \times 2$ MIMO system when the optical power level in both paths at cores $\mathrm{c} 1$ and $\mathrm{c} 2$ is reduced simultaneously, $+2 \mathrm{~dB}$ received power is required compared with DL SISO, as the 3GPP EVM recommendation of the $2 \times 2$ MIMO LTE-A signal is met for $P_{\text {PIN }} \geq-8 \mathrm{dBm}$ while doubling the user bitrate compared with its SISO counterpart over the same bandwidth.

Fig. 6(b) includes the EVM results obtained when reducing only the master path in the $2 \times 2$ MIMO signal (using the VOA at the receiver of the optical path of core c1 in Fig. 4) while keeping the optical power level of core $\mathrm{c} 2$ at $\mathrm{P}_{\mathrm{PIN}} \mathrm{c} 2=-5 \mathrm{dBm}$. We observe that the performance tends to be similar to a DL SISO for $\mathrm{P}_{\mathrm{PIN}} \leq-8 \mathrm{dBm}$. We evaluate this behavior in more detail in Fig. 7 by analyzing the quality of the reference signal over each receiver antenna, also known as C-RS/Rx paths. In the case of $2 \times 2$ MIMO transmission depicted in Fig. 4, the $\mathrm{C}-\mathrm{RS}$ port 0 and $\mathrm{Rx} 0(\mathrm{C}-\mathrm{RS} 0 / \mathrm{Rx} 0)$ corresponds to the signal of core $\mathrm{c} 1$ (master) while C-RS1/Rx1 corresponds to the signal coming from core $\mathrm{c} 2$ (slave). We can observe in Fig. 7(a) that, when the power of both paths $\mathrm{c} 1$ and $\mathrm{c} 2$ is reduced simultaneously, the quality of the C-RS0/Rx0 and C-RS1/Rx1 have similar performance with the received power.
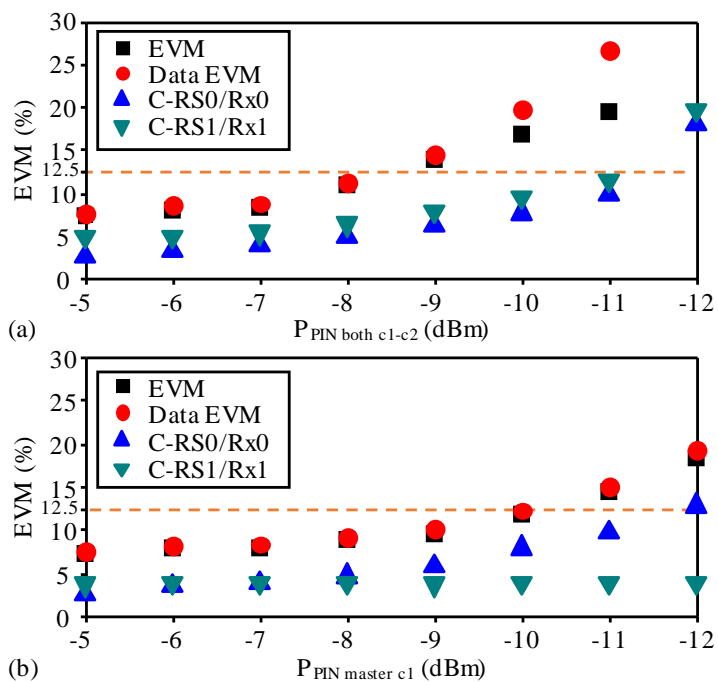

Fig. 7. Measured EVM of CCO in a two-antenna LTE-A system implementing $2 \times 2$ MIMO including the reference signal quality over each receiver antenna when we reduce the optical power level of (a) both master and slave signals simultaneously (cores $\mathrm{c} 1$ and $\mathrm{c} 2$ ) or (b) only master signal in core $\mathrm{c} 1$.

But when we only reduce the master signal in core $\mathrm{c} 1$-Fig. 7(b)- only the C-RSO/Rx0 is affected, while the quality of the C-RS1/Rx1 remains more or less constant as the slave signal travelling by core $\mathrm{c} 2$ of the MCF is not reduced ( $\mathrm{P}_{\mathrm{PIN}} \mathrm{c} 2=$ $-5 \mathrm{dBm})$. Obviously, the measured EVM and data EVM is also affected as it takes into account both data streams of the MIMO signal. This confirms the MIMO capability to compensate the quality of one path with another in the $2 \times 2$ matrix multiplex. Having a closer look at the results compared in Fig. 6(b) we observe that, when $2 \times 2$ MIMO is used and we reduce only the power level of the master path at core $\mathrm{c} 1$, the LTE-A signal meets the EVM recommendation for $P_{P I N c 1} \geq$ $-10 \mathrm{dBm}$, as the MIMO processing is able to compensate the quality of the signal received from core $\mathrm{c} 1$ with the signal coming from core $\mathrm{c} 2$ with $\mathrm{P}_{\mathrm{PIN} \mathrm{c} 2}=-5 \mathrm{dBm}$. This is interesting for the definition of the RoF system as provides an extra $2 \mathrm{~dB}$ margin in the power balance between the two cores of the MCF media to achieve the same performance as SISO but providing double user bitrate over the same bandwidth.

\section{Four-Antenna $4 \times 4$ MIMO LTE-A radio-over-multicore fiber transmission performance}

Using the same procedure and with the experimental setup depicted in Fig. 8 for a four-antenna system implementing $4 \times 4$ MIMO spatial multiplexing, we use the VOAs to reduce the optical power level arriving at the photodiodes $\left(\mathrm{P}_{\mathrm{PIN}}\right)$ of a master or a slave signal.

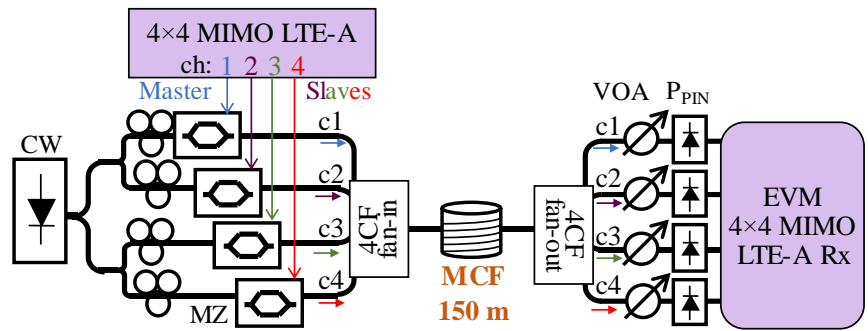

Fig. 8. Experimental setup for the evaluation of four-antenna LTE-A system implementing 4×4 MIMO spatial multiplexing over MCF 
Fig. 9(a) shows the performance of the DL physical channels -depicted in Table 1- of the component carrier CC0 (located at $f_{c}-9.9 \mathrm{MHz}$ ) after MCF transmission when the optical power of the master signal is reduced (path in core $\mathrm{c} 1$ ) and the slave signals remain constant (paths in cores $\mathrm{c} 2, \mathrm{c} 3$ and c4). We can observe that, when the power level of an optical path level is reduced, the primary and secondary synchronization signal channels P-SS and S-SS are deeply affected. It can be observed in Fig. 9(a) that the performance of the cell-specific reference signal (C-RS) also degenerates with the power level until $\mathrm{P}_{\text {PIN Master } \mathrm{c} 1} \leq-17 \mathrm{dBm}$ when the reference signal is not found, as confirmed by the constellation depicted in Fig. 10(c).
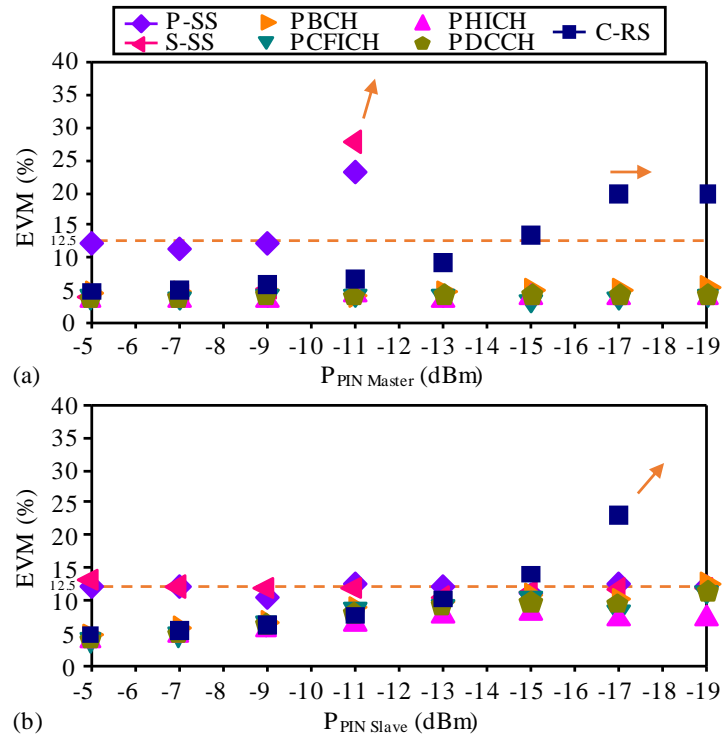

Fig. 9. EVM performance evaluation of $\mathrm{CC} 0$ physical channels in a fourantenna LTE-A system implementing $4 \times 4$ MIMO over a four-core MCF when the optical power of (a) the master signal (core $\mathrm{c} 1$ ) and (b) the slave signal (core c3) is reduced, while the other optical paths remain constant.
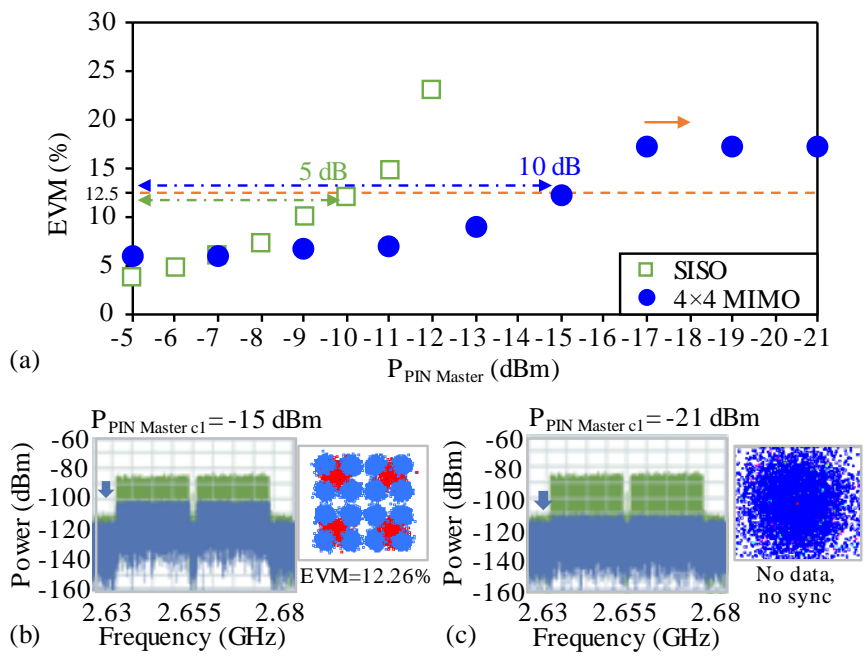

Fig. 10. (a) Comparison of $\mathrm{CC} 0$ performance in a single-antenna and a fourantenna LTE-A system implementing $4 \times 4$ MIMO over a four-core MCF when the optical power of the master signal (core $\mathrm{c} 1$ ) is reduced and the other optical paths remain constant. Measured spectrum $(\mathrm{RBW}=1.27 \mathrm{kHz})$ and constellation of Layer 0 of $\mathrm{CC} 0$ for constant slaves signals and reduced master with (b) PPIN Master $=-15 \mathrm{dBm}$ and (c) PPIN Master $=-21 \mathrm{dBm}$.
Fig. 10(a) shows the performance comparison of a singleantenna and a four-antenna system when the master signal is reduced. We can observe in the constellation included in Fig. 10(c) that when the received power of the optical path in core c1 is lower than PPIN Master $\leq-17 \mathrm{dBm}$ the synchronization is lost. Fig. 11 shows the experimental performance obtained when the master and two slaves remain constant (optical paths in cores $\mathrm{c} 1, \mathrm{c} 2$ and $\mathrm{c} 4$ ) and only the slave in the optical path of core $\mathrm{c} 3$ is reduced. The experimental results confirm the standard LTE-A wireless behavior as, when the path affected corresponds to a slave signal, for low optical power levels the synchronization from the master signal is present but no data is demodulated at the LTEA receiver. The change in the received constellations can be observed in Fig. 11(b)-(c) where only the synchronization signal is received for PPIN Slave c $3 \leq-21 \mathrm{dBm}$.

As represented in both Fig. 10(a) and Fig. 11(a), a singleantenna LTE-A system (SISO) meets the EVM requirements for optical power levels at the receiver ranging from $-5 \mathrm{dBm}$ to $-10 \mathrm{dBm}$. This provides a $5 \mathrm{~dB}$ power variation margin in the optical power budget to meet the EVM requirements at the receiver.

In comparison, a four-antenna LTE-A system implementing $4 \times 4$ MIMO spatial multiplexing over MCF is received within the $3 \mathrm{GPP}$ EVM recommendation for $\mathrm{P}_{\mathrm{PIN} \text { Master }} \geq-15 \mathrm{dBm}$, as confirmed by the constellations shown in Fig. 10(b) and Fig. 11(b). This provides a $10 \mathrm{~dB}$ power margin between the four paths (between $-5 \mathrm{dBm}$ and $-15 \mathrm{dBm}$ ) which is $+5 \mathrm{~dB}$ higher than with SISO.

This confirms the suitability of using the in-built 3GPP MIMO algorithms for the transmission of LTE-A over MCF, which enables providing almost four times the bitrate of a single-antenna system over the same bandwidth with $5 \mathrm{~dB}$ extra power margin between the optical paths.
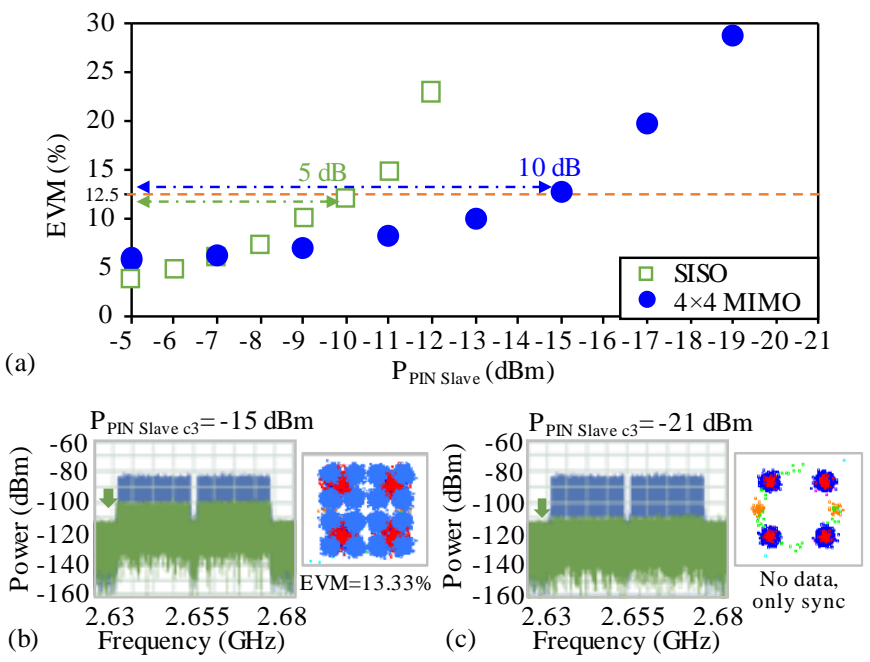

Fig. 11. (a) Comparison of $\mathrm{CC} 0$ performance in a single-antenna and a fourantenna LTE-A system implementing $4 \times 4$ MIMO over a four-core MCF when the optical power of the slave signal (core c3) is reduced and the other optical paths remain constant. Measured spectrum $(\mathrm{RBW}=1.27 \mathrm{kHz})$ and constellation of Layer 0 of $\mathrm{CC} 0$ for constant master/slaves and reduced slave signal at core $\mathrm{c} 3$ with (c) PPIN Slave $\mathrm{c} 3=-15 \mathrm{dBm}$ and (d) PPIN Slave $\mathrm{c} 3=-21 \mathrm{dBm}$. 


\section{CARRIER-AGGREgATION PERFORMANCE IN FOUR- ANTENNA 4×4 MIMO LTE-A SYSTEMS OVER MCF}

In this section, we analyze the performance of a four-antenna LTE-A system implementing 4×4 MIMO spatial multiplexing of four data streams for a single user as depicted in Fig. 2(d). Using the same laboratory setup employing the four channel outputs and four channel inputs of the wireless test described in Fig. 8, we evaluate the performance of LTE-A signal implementing 4×4 MIMO spatial multiplexing and Carrier Aggregation for different frequency bands $\left(f_{c}\right)$ and different carrier separation $(\Delta f)$.

Fig. 12(a) shows the EVM of both aggregated carriers for different carrier separation ( $\Delta f)$ maintaining the center frequency at $f_{c}=2.655 \mathrm{GHz}$ (center of FDD band 7).

Comparing the measured spectrum in Fig. 12(b)-(d), for $\Delta f= \pm 9.1 \mathrm{MHz}$ the $20 \mathrm{MHz}$ carriers start overlapping in spectrum which increases the EVM to 8.16\% compared with the EVM of $6.76 \%$ obtained with $\Delta f= \pm 10.3 \mathrm{MHz}$.

Fig. 13(a) shows the experimental results obtained for the $4 \times 4$ MIMO LTE-A RoF performance for different center frequencies $\left(f_{c}\right)$, including the middle of regulated and commercially available downlink FDD band 20 $\left(f_{c}=804 \mathrm{MHz}\right)$ and band $7\left(f_{c}=2.655 \mathrm{GHz}\right)$. As it can be observed in Fig. 13(a), the frequency response of the optoelectrical conversion degenerates the EVM after MCF transmission from $3.87 \%$ at $0.5 \mathrm{GHz}$ to $7.76 \%$ at $3 \mathrm{GHz}$.
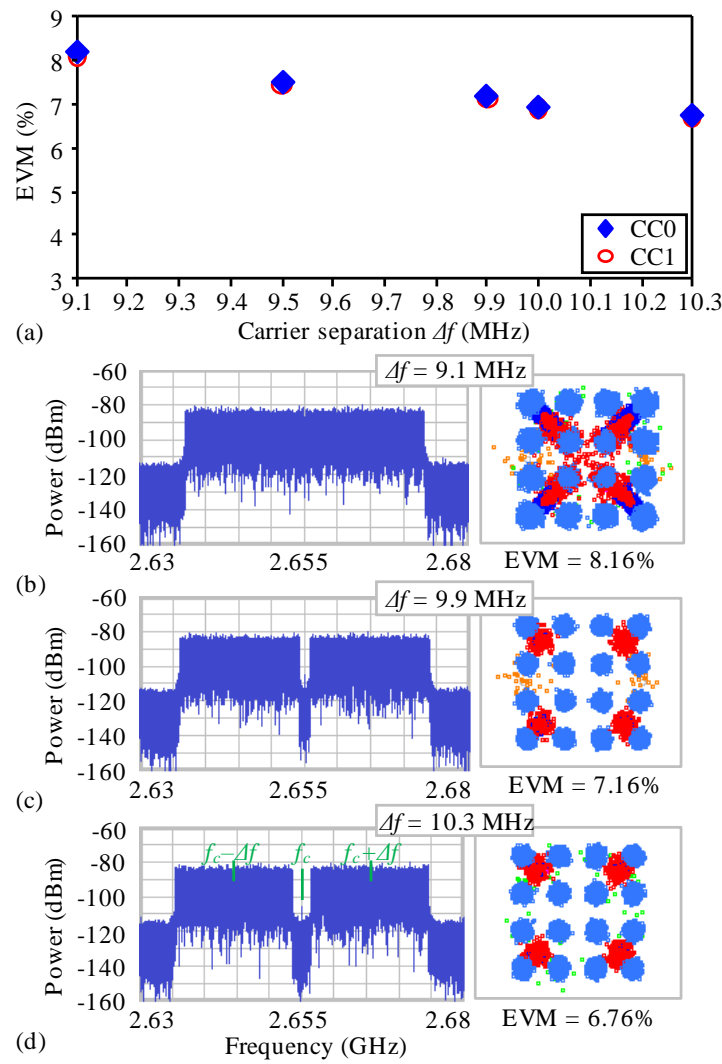

Fig. 12. (a) Measured EVM for two $20 \mathrm{MHz}$ LTE-A $4 \times 4$ MIMO CCs vs. carrier separation and measured spectrum (ch1, $\mathrm{R}_{\mathrm{BW}}=1.27 \mathrm{kHz}$ ) and constellations for (b) $\Delta f=9.1 \mathrm{MHz}$, (c) $\Delta f=9.9 \mathrm{MHz}$, and (d) $\Delta f=10.3 \mathrm{MHz}$
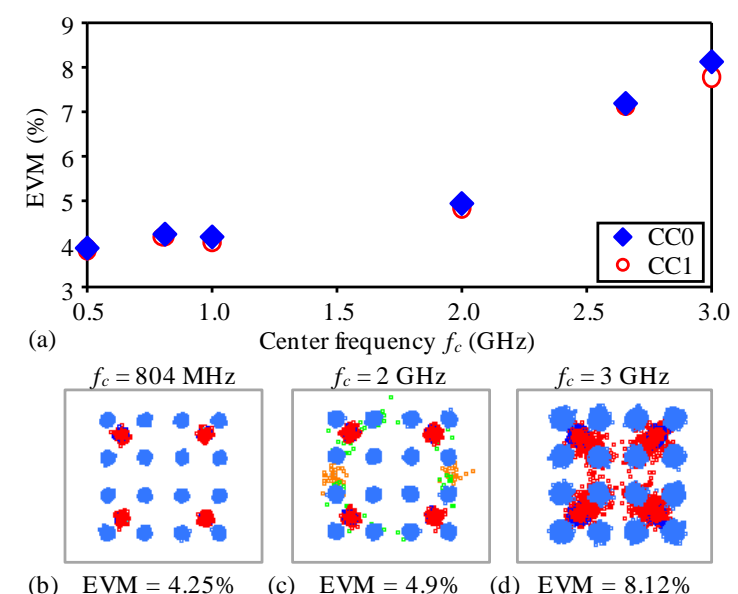

Fig. 13. (a) Measured EVM for two $20 \mathrm{MHz}$ LTE-A $4 \times 4$ MIMO CCs with $\Delta f= \pm 9.9 \mathrm{MHz}$ vs. center frequency and constellations for (b) $f_{c}=804 \mathrm{MHz}$, (c) $f_{c}=2 \mathrm{GHz}$, and (d) $f_{c}=3 \mathrm{GHz}$

The results reported in Fig. 13(a) confirm that the EVM performance is similar for both aggregated carriers separated $\Delta f= \pm 9.9 \mathrm{MHz}$. Fig. 13(b)-(d) show some examples of the received constellations and measured EVM of the data coded in Layer 0 of the carrier $\mathrm{CC} 0$ for different center frequencies. These results confirm that Carrier Aggregation does not affect the signal quality as long as the CCs are not overlapped in spectrum, but the frequency response of the electro-optical devices should be taken into account depending on the $3 \mathrm{GPP}$ band used.

\section{CONCLUSION}

This paper proposes and evaluates experimentally the performance of multiple-antenna RoF fronthaul over MCF media implementing MIMO and Carrier Aggregation. Different configurations of single- and multiple-antenna systems can be developed assigning one or a group of cores of the MCF to each system. In this work we evaluated a singleantenna LTE-A system in both DL and UL configurations, a two-antenna LTE-A system implementing $2 \times 2$ MIMO and a four-antenna LTE-A system implementing $4 \times 4$ MIMO spatial multiplexing. The experimental evaluation is performed including also Carrier Aggregation with two carriers at the maximum regulated bandwidth. The experimental results confirm that Carrier Aggregation doesn't affect the signal quality as long as the carriers are not overlapped in spectrum. More carriers could be provided in the same or different frequency bands employing the proposed radio-over-multicore fiber fronthaul architecture, but taking into account the frequency response of the electro-optical devices depending on the $3 \mathrm{GPP}$ band used.

The evaluation of single-antenna systems indicates that LTE-A DL signals have better performance after RoF than UL signals. In this case, with the RoF over $150 \mathrm{~m}$ of MCF the single-antenna (SISO) LTE-A UL signal requires an extra $+1 \mathrm{~dB}$ received optical power level than the single-antenna LTE-A DL configuration ( $\mathrm{P}_{\mathrm{PIN}} \geq-10 \mathrm{dBm}$ ). Using $2 \times 2 \mathrm{MIMO}$ spatial multiplexing provides double user bitrate over the same 
bandwidth than SISO with only $+2 \mathrm{~dB}$ received power penalty $\left(P_{\text {PIN }} \geq-8 \mathrm{dBm}\right)$ The experimental results point out that the quality of the spatially multiplexed MIMO signal is an average of the multiplexed data streams. This can be used to compensate the quality of one path with another. When $2 \times 2$ MIMO is implemented and only the power level of the master path is reduced, the LTE-A signal meets the EVM recommendation for $P_{\mathrm{PIN}} \geq-10 \mathrm{dBm}$, overcoming the $2 \mathrm{~dB}$ power penalty mentioned before. This processing benefit is even more significant in a four-antenna LTE-A system implementing $4 \times 4$ MIMO spatial multiplexing over MCF. In this case, the balance between the four streams can compensate the signal with a path receiving optical power levels of as low as $P_{\mathrm{PIN}} \geq-15 \mathrm{dBm} .4 \times 4$ MIMO processing provides a $+5 \mathrm{~dB}$ power variation margin between cores to meet the EVM requirements at the receiver compared with its SISO counterpart while increasing $\times 4$ the user bitrate over the same bandwidth. This experimental evaluation confirms the successful transmission of carrier-aggregated LTE-A signals employing the 3GPP MIMO processing algorithms already implemented in commercial devices for the MCF transmission in fronthaul applications. The proposed architecture can be scaled to massive MIMO deployment scenarios considering large core-count MCF already available in the market.

\section{REFERENCES}

[1] M. Selim et al., "Fronthaul cell outage compensation for 5G networks," IEEE Communications Magazine, vol. 54, no. 8, pp. 169-175, 2016

[2] T. Kanesan et al., "Solution to reduce nonlinearity in LTE RoF system for an efficient DAS topology: A brief review," in Proc. Int. Symp. on Comm. Systems, Networks and Digital Signal Processing (CSNDSP), July 2016.

[3] A.A. Ali et al., "Investigation of user scheduling schemes under different MIMO transmission modes for carrier aggregation in LTE-A," in Proc. 2014 IEEE International Symposium on Telecommunication Technologies (ISTT), pp.268-272, Nov. 2014.

[4] Gee-Kung Chang, Cheng Liu, and Liang Zhang, "Architecture and applications of a versatile small-cell, multi-service cloud radio access network using radio-over-fiber technologies," in Proc. EEE International Conference on Communications (ICC), pp. 879-883, June 2013.

[5] Jun-ichi Kani, "Solutions for future mobile fronthaul and accessnetwork convergence," in Proc. Optical Fiber Communications Conference and Exhibition (OFC), paper W1H.1, March 2016.

[6] H. Q. Ngo, E. G. Larsson, and T. L. Marzetta, "Energy and spectral efficiency of very large multiuser MIMO systems," IEEE Trans. Commun., vol. 61, no. 4, pp. 1436-1449, Apr. 2013.

[7] M. Morant, A. Macho and R. Llorente, "On the suitability of multicore fiber for LTE-Advanced MIMO optical fronthaul systems," IEEE/OSA Journal of Lightwave Technology, vol. 34, no. 2, pp. 676-682, January 2016.

[8] Jun-ichi Kani, "Solutions for future mobile fronthaul and accessnetwork convergence," in Proc. Optical Fiber Communications Conference and Exhibition (OFC), pp. 1-43, March 2016.

[9] J. Gozalvez, "New 3GPP Standard for IoT [Mobile Radio]," IEEE Vehicular Technology Magazine, vol. 11, no. 1, pp. 14-20, March 2016.

[10] C-P. Liu and A. J. Seeds, "Transmission of wireless MIMO-type signals over a single optical fiber without WDM," IEEE Transactions on Microwave Theory and Techniques, vol. 58, mo. 11, pp. 3094-3102, 2010.

[11] M. Chen, X. Xiao, J. Yu, X. Li, F. Li, "Real-time gigabit RS-coded OFDM signal transmission over WDM-based X-band $2 \times 2$ MIMO RoF system," in Proc. Optical Fiber Communications Conference and Exhibition (OFC), paper Th4E.3, 2017.
[12] J. He, B. Li, L. Deng, M. Tang, L. Gan, S. Fu, P. Ping Shum and D. Liu, "Experimental demonstration of bidirectional OFDM/OQAM-MIMO signal over a multicore fiber system," IEEE Photonics Journal, vol. 8, no. 5,2016

[13] T. Kanesan, S. Rajbhandari, E. Giacoumidis, I. Aldaya, "Nonlinear limit of alternative method to 2 × 2 MIMO for LTE RoF system," Electronics Letters, vol. 50, no. 4, pp. 300-301, 2014.

[14] S. L. Jansen, I. Morita, H. Tanaka, "16x52.5-Gb/s, 50-GHz spaced, POLMUX-CO-OFDM transmission over 4,160 km of SSMF enabled by MIMO processing," in Post-Deadline Proc. 33rd European Conference and Exhibition of Optical Communication (ECOC), 2008.

[15] Yumeng Yang, Michael J. Crisp, Richard V. Penty, Ian H. White, "Low-cost MIMO radio over fiber system for multiservice DAS using double sideband frequency translation," IEEE/OSA Journal of Lightwave Technology, vol. 34, no. 16, pp. 3818-3824, 2016

[16] M. Morant and R. Llorente, "Experimental demonstration of LTE-A $\mathrm{M} \times 4 \times 4$ MIMO radio over multicore fiber fronthaul," in Proc. Optical Fiber Communications Conference and Exhibition (OFC), p. Th4E.4, March 2017.

[17] T. Sakamoto, S. Aozasa, T. Mori, M. Wada, T. Yamamoto, S. Nozoe, Y. Sagae, K. Tsujikawa, K. Nakajima, "Twisting-rate-controlled $125 \mu \mathrm{m}$ cladding randomly-coupled single-mode 12-core fiber," IEEE/OSA Journal of Lightwave Technology, vol. PP, 2017.

[18] Keysight Technologies, Inc., "LTE-Advanced physical layer overview," RF \& Microwave Navigation Map, 2017 [Online]. Available: http://rfmw.em.keysight.com/wireless/helpfiles/89600B/WebHelp/Subsy stems/lte-a/content/lte_overview.htm

Maria Morant (S'07-M'12) received the M.Sc. degree in telecommunication engineering and the International Ph.D. degree from the Universitat Politècnica de València, Valencia, Spain, in 2008 and 2012, respectively. Since 2006, she has been investigating optical techniques for the transmission of OFDM-based signals in access networks at Valencia Nanophotonics Technology Center (NTC). She is currently a postdoc researcher at NTC working in advanced optical sensing and next-generation MIMO communications.

She has participated in European projects dealing with optical communications and optical sensing such as FP7-ICT-FIVER, FP7-ISTUCELLS and FP6-IST-UROOF and in national projects like ULTRADEF, VISICONEC and HIDRASENSE. She has contributed with more than 75 papers to international journals and hot-topic conferences on optical communications. Her current research interests include advanced modulations and optical sensing techniques.

Roberto Llorente (S'99-S'06) received the M.Sc. degree in telecommunication engineering from the Universitat Politècnica de València (UPV), Valencia, Spain and the European Ph.D. degree from the same university in 2006. Since then, he has been in research positions within the university, and in 2002 he joined the Valencia Nanophotonics Technology Center (NTC) as a Research Associate. He is currently a Professor in the UPV teaching Optical Communications related subjects. He is also the Head of the Optical Systems and Networks Unit in the NTC. He has been leading NTC activities in the European projects FP5-IST-TOPRATE, FP6-IST-UROOF between others. He has proposed and coordinated FP7-IST-UCELLS and FP7-ICT-FIVER projects in the field of radio-over-fiber technology and nextgeneration FTTH networks. He is currently leading NTC activities in national and international research projects dealing with optical signal processing applied to optical networks and next-generation test \& measurement.

$\mathrm{He}$ has authored or co-authored more than 150 papers in leading international journals and conferences and has authored three patents. His current research interests include optical and electro-optical processing applied to optical core and metro networks, hybrid wireless-optical access networks, optical sensing, metrology, and medical imaging systems. 